\title{
LA CIENCIA VISTA DESDE LA DIVERSIDAD CULTURAL: CONSIDERACIONES PARA UN CURRÍCULO EN PROGRAMAS DE FORMACIÓN INICIAL DE CIENCIAS NATURALES Y EDUCACIÓN AMBIENTAL
}

\section{A CIÊNCIA VISTA A PARTIR DA DIVERSIDADE CULTURAL: CONSIDERAÇÕES PARA UM CURRÍCULO EM PROGRAMAS DE FORMAÇÃO INICIAL EM CIÊNCIAS DA NATUREZA E EDUCAÇÃO AMBIENTAL}

\begin{abstract}
RESUMEN: Este artículo propone algunas consideraciones curriculares para Programas de Formación Inicial de Profesores, con base en la interpretación de resultados de la Tesis Doctoral denominada "Concepciones de ciencia desde la perspectiva de la diversidad cultural en docentes de programas de Licenciatura en Ciencias Naturales y educación Ambiental en Colombia - PFILCNYEA". Inicialmente aborda el posicionamiento de la episteme curricular fundamentada desde las epistemologías del sur, la cuales se basan en las ecologías del conocimiento y la traducción intercultural. De otra parte, como principios se plantean: la diversidad cultural como proyecto educativo, social y político. En relación a las estrategias se propone a la diversidad cultural como eje fundante y reflexivo del currículo de PFILCNYEA, en relación a los objetivos de los planes de estudio, organización de las mallas académicas y evaluación. Finalmente, se indican perspectivas sobre la implementación de un currículo que conciba a la ciencia desde la diversidad cultural para la formación inicial de profesores en Colombia como país pluriétnico y multicultural.
\end{abstract}

Palabras clave: Concepciones de Ciencia; Diversidad cultural; Enseñanza de las ciencias; Currículo; Formación inicial de profesores.

RESUMO: Este artigo propõe algumas considerações curriculares para os Programas de Formação Inicial de Professores a partir da interpretação dos resultados da tese de doutorado denominada Concepções de ciência na perspectiva da diversidade cultural em professores de bacharelado em Ciências Naturais e Programas de Educação Ambiental em. Colômbia - PFILCNYEA. Inicialmente, aborda o posicionamento da episteme curricular a partir das epistemologias do sul, que se fundamentam nas ecologias do conhecimento $e$ da tradução intercultural. Por outro lado, os princípios são: a diversidade cultural como projeto educacional, social e político. Em relação às estratégias, a diversidade cultural é

\footnotetext{
Doutorando em Educação pela Universidade Distrital Francisco José de Caldas (Colômbia). Contato: juadame@areandina.edu.co
} 
proposta como eixo fundador e reflexivo do currículo PFILCNYEA, em relação aos objetivos dos planos de estudos, organização das redes acadêmicas e avaliação. Por fim, são indicadas perspectivas sobre a implementação de um currículo que conceba as ciências a partir da diversidade cultural para a formação inicial de professores na Colômbia como um país multiétnico e multicultural.

Palavras-chave: Concepções de ciencia; Diversidade cultural; Ensino de ciencias; Currículo; Formação inicial de professores.

\section{INTRODUCCIÓN}

Una educación descontextualizada, la cual no reconoce el territorio en donde se desarrolla y desconoce su historia, su política o su cultura, pueda arraigarse como una grave problemática que repercuta en la disminución de la significancia o de legitimidad del conocimiento para el estudiantado (WALSH, 2012). En este sentido, la ciencia occidental pareciera verse como único fin de la educación científica (VÁZQUEZ; ACEVEDO; MANASSERO; ACEVEDO, 2001). Este aspecto podría entenderse como una de las principales dificultades contemporáneas de la enseñanza de las ciencias y la formación de su profesorado, teniendo en cuenta que el aula se concibe como un espacio heterogéneo y que el acto educativo se consolida a través de la diversidad en la misma, conllevando a replantear su imagen a través de las diferentes concepciones vinculadas a creencias, cosmovisiones, cosmogonías o epistemologías propias del estudiantado (SÁNCHEZ-ARTEAGA; SEPÚLVEDA; EL-HANI, 2013).

Desde el fenómeno actual de la modernidad y la globalización, la sociedad representa un escenario de diversidad cultural, presentándose como una mixtura de grupos étnicos que subyacen a distintos procesos migratorios, de desplazamientos y diásporas; sin embargo, pareciera que diferentes factores que se ciñen a tendencias económicas y políticas mundiales, están conllevando a la homogenización del conocimiento desde la occidentalización del mismo, afectando a países que poseen un gran acervo cultural a través de la desaparición de sus comunidades ancestrales (URIBE, 2019). 
Trabajos como los de Adame (2020), vislumbran a los Programas de Formación Inicial de Licenciados en Ciencias Naturales en Educación Ambiental en Colombia sumergidos ante un posicionamiento del conocimiento único y globalizado, es decir, la ciencia se consolida como el centro epistemológico docente preponderante, en donde parece aún prevalecer entre maestros e investigadores una diferenciación entre los conocimientos científicos y las concepciones denominadas alternativas, sobrevalorando el conocimiento producto de la ciencia frente a otras diversos saberes, hecho que parece sostenerse en el tiempo dentro de la historia educativa y político-escolar de nuestra nación.

En relación a todo lo descrito anteriormente, este artículo busca dar respuesta a la siguiente pregunta: ¿cuáles consideraciones deben optarse para desarrollar un currículo orientado a la formación inicial de profesores de ciencias naturales y educación ambiental, que conciba la ciencia desde la diversidad cultural?

\section{POSICIONAMIENTO EPISTEMOLÓGICO: INTRODUCCIÓN DE LAS EPISTEMOLOGÍAS DEL SUR COMO FUNDAMENTO DE LA DIVERSIDAD CULTURAL EN EL CURRÍCULO DE LOS PROGRAMAS DE FORMACIÓN INICIAL DE PROFESSORES DE CIENCIAS}

América Latina es una consecuencia y un producto de la geopolítica del conocimiento impuesto por la "modernidad", la cual es entendida como un proceso de cambios que buscan homogeneizar a la sociedad, a través de la apropiación de concepciones y explicaciones de fenómenos desde un entendimiento universal (MIGNOLO, 2003). Lander (2000) aborda la idea de modernidad a partir de cuatro dimensiones, la primera hace referencia a la visión universal de la historia asociada a la idea del progreso por la cual se construye la clasificación y jerarquización de todos los pueblos y continentes desde una postura eurocéntrica. La segunda comprende el concepto de "naturalización" tanto de las relaciones sociales como de la naturaleza humana, la cual asume una condición empírica investida a través de un diálogo entre la teoría del conocimiento y las ciencias de la cognición. La tercera, 
la naturalización u ontologización de las múltiples separaciones propias de la sociedad, y la cuarta relata la superioridad de los conocimientos que produce esa sociedad (científicos) sobre otros tipos de saberes (propios, tradicionales y/o ancestrales).

Así pues, la naturalización del ejercicio de la violencia social en nuestro tiempo es impulsada a través de múltiples prácticas discursivas, propagadas en cierta manera a través de la manipulación del conocimiento y la relación del Estado y sus políticas para contener, revertir y desarticular lo colectivo y tal vez diverso (PALERMO, 2014).

El término más pujante de la eficacia del pensamiento científico moderno especialmente en sus dicciones tecnocráticas hoy hegemónicas, puede describirse literalmente como la naturalización de las relaciones sociales, es decir, nos encontramos ante una sociedad sin ideologías, que converge ante un modelo civilizatorio único, globalizado, con una postura hegemónica del pensamiento, el cual presenta su propia narrativa histórica como el conocimiento objetivo, científico y universal, lográndose interpretar dentro de la sociedad moderna como el más avanzado, no siendo otra cosa más que la colonización del saber (LANDER, 2000). Entonces, asumimos que la "modernidad" es producto de una relación colonial de dominio (DUSSEL, 1994).

De tal forma, las corrientes hegemónicas de la modernidad en Latinoamérica han conllevado la sumisión e inequidad cultural en relación a la preponderación del conocimiento científico occidental frente a los saberes propios, por consiguiente, es importante fragmentar estas segmentaciones, posibilitando procesos de inclusión, reconocimiento y legitimación de los mismo ante la sociedad y la educación (URIBE, 2019). Lo anterior, nos lleva a deliberar sobre una lógica cultural de herencias coloniales, que subsisten en nuestras subjetividades y en nuestras posturas de enfrentar la realidad, las cuales son en gran parte, representadas como condiciones aprendidas e interiorizadas de negación de nuestro ser, nuestro saber y nos ubican social y políticamente bajo el yugo de una inferioridad permanente y naturalizada. Lo descrito, nos lleva a pensar sobre una corriente de pensamiento decolonial 
fundamentada sobre investigaciones como las de QUIJANO (2000), MIGNOLO (2003), WALSH (2010) y LANDER (2000), que implica reconocer la herencia de las diferentes colonialidades, sin llegar a desconocer nuestros ideales, proponiendo entonces, la construcción de nuestro proyecto emancipatorio como pueblos latinoamericanos que poseen sus propias racionalidades de vida en disposición al diálogo crítico intercultural con otras lógicas de mundo, ante un sistema global y una ciencia moderna que ha demostrado su incapacidad como propuesta humanizante y universal (GÓMEZ et al., 2014).

De acuerdo con lo mencionado, los programas de formación de profesores deberían incorporar una reflexión decolonial, en cuanto a la concepción de distintos saberes en el ámbito académico, favoreciendo una imagen de ciencia que le permita comprender y consolidar su acto educativo desde la diversidad cultural. Esta irrupción en el escenario de la educación superior en nuestro país, permite reivindicar gran variedad y multiplicidad de subjetividades que históricamente han sido invisibilizadas por la introducción apológica del conocimiento científico en una nación pluriétnica y multicultural, buscando entonces emancipar nuevos discursos y realidades desde la academia, que en otros términos significa la promoción de saberes contrahegemónicos a través de la aproximación a epistemologías decoloniales o del Sur (NIETO, 2014). Entonces, las epistemologías del Sur contribuyen al reconocimiento de las comunidades excluidas, favoreciendo una crítica al paradigma científico dominante, al tiempo que permiten el diálogo con otras epistemologías.

Teniendo en cuenta a Sousa Santos (2011), las epistemologías del Sur se basan en la sociología de las ausencias y las emergencias, el pensamiento posabismal y el buen vivir. Se fundamenta en dos premisas, la primera orientada a la comprensión del mundo, siendo esta mucho más amplia que la comprensión occidental del mundo, la segunda hace referencia en que la diversidad del mundo es infinita, la cual incluye modos muy distintos de ser, pensar y sentir, de concebir el tiempo, la relación entre seres humanos y entre humanos y no humanos, de mirar 
el pasado y el futuro, de organizar colectivamente la vida, la producción de bienes y servicios y el ocio (SOUSA SANTOS, 2011).

La ecología de saberes comienza con la asunción de que todas las prácticas de relaciones entre los seres humanos, así como entre los seres humanos y la naturaleza, implican más de una forma de conocimiento y, por ello, de ignorancia. Epistemológicamente, la moderna sociedad capitalista se caracteriza por el hecho de que favorece prácticas en las que predomina el conocimiento científico (SOUSA SANTOS, 2011, p. 9).

La segunda idea central es la traducción intercultural, comprende como el procedimiento permite crear inteligibilidad recíproca entre las experiencias del mundo, tanto las disponibles como las posibles, sin atribuir un estatuto de totalidad exclusiva ni el de parte homogénea. Las experiencias del mundo son tratadas en momentos diferentes del trabajo de traducción como totalidades o partes y como realidades que no se agotan en esas totalidades o partes.

\section{PRINCIPIOS FUNDANTES DE UN CURRÍCULO PARA LA FORMACIÓN DE PROFESORES DE CIENCIA DESDE LA DIVERSIDAD CULTURAL}

La diversidad cultural como proyecto educativo

Una de las principales causas de la diversidad cultural presentes en las escuelas colombianas es el fenómeno migratorio que se ha producido en los últimos años como consecuencia principalmente a las diásporas fruto de la pobreza, violencia, guerra, globalización y mercado laboral, en este sentido, debemos reconocer que esto hace parte de la cotidianidad y el aula de clases es fiel reflejo de los cambios que suceden en nuestra sociedad actual.

Entonces, la diversidad pone en evidencia en estos espacios académicos diferentes expresiones e identidades culturales propias de un pueblo, región, país, continente y del mundo entero, implicando el contacto entre diversas etnias, lenguas, religiones, expresiones artísticas, valores, tradiciones, prácticas y cosmovisiones (URIBE, 2019). 
La Organización de las Naciones Unidas para la Educación, la Ciencia y la Cultura (UNESCO, 2005), define a la diversidad cultural como la multiplicidad de formas en que se expresan las culturas de los grupos y sociedades, enriqueciendo y transmitiendo el patrimonio cultural de la humanidad mediante la variedad de expresiones culturales, a través de distintos modos de creación artística, producción, difusión, distribución y disfrute de expresiones. Desde el marco histórico de la educación que tradicionalmente ha sido impartida desde la occidentalización de conocimiento en la escuela, nace una reflexión a la diversidad en relación a las minorías, esto, por necesitar un trato conveniente por la brecha cultural representada por la identidad dominante frente a las mismas, y los fracasos asociados del estudiantado cuando acceden a esta. En relación a lo anterior, investigaciones como las de García, Pulido y Montes (1997), proponen algunos modelos que han permitido aproximar una educación multicultural en la escuela:

Tabla 1. Modelos de Educación Multicultural

\begin{tabular}{|c|c|c|}
\hline AUTORES & MODELO & PROPOSITO \\
\hline $\begin{array}{l}\text { Sleeter \& } \\
\text { Grant } \\
(1988)\end{array}$ & $\begin{array}{l}\text { Educar para } \\
\text { igualar: la } \\
\text { asimilación } \\
\text { cultural }\end{array}$ & $\begin{array}{l}\text { Igualar las oportunidades educativas para alumnos } \\
\text { culturalmente diferentes. La educación multicultural, que desde } \\
\text { esta perspectiva evita los supuestos de esos programas de } \\
\text { compensatoria que niegan las diferencias culturales, y asumen } \\
\text { una patología del ambiente familiar e intenta cambiar a los } \\
\text { niños, su lengua e, incluso, las pautas de sus padres sobre la } \\
\text { crianza. }\end{array}$ \\
\hline $\begin{array}{l}\text { García, } \\
\text { 1978; } \\
\text { Seifer, } \\
\text { 1973; } \\
\text { Wynn, } \\
\text { 1974; } \\
\text { Solomon, } \\
\text { 1988; } \\
\text { Sleeter \& } \\
\text { Grant } \\
\text { 1988) }\end{array}$ & $\begin{array}{c}\text { El } \\
\text { entendimiento } \\
\text { cultural: el } \\
\text { conocimiento } \\
\text { de la diferencia }\end{array}$ & $\begin{array}{l}\text { Se apuesta por una necesaria educación acerca de las } \\
\text { diferencias culturales y no de una educación de los llamados } \\
\text { culturalmente diferentes. Se trata de enseñar a todos a valorar } \\
\text { las diferencias entre las culturas. Hay que preparar a los } \\
\text { estudiantes para que vivan armoniosamente en una sociedad } \\
\text { multiétnica, y para ello habrá que abordar en el aula las } \\
\text { diferencias y similitudes de los grupos, con objeto de que los } \\
\text { alumnos comprendan esa pluralidad }\end{array}$ \\
\hline $\begin{array}{l}\text { (Stickel, } \\
\text { 1987) }\end{array}$ & $\begin{array}{l}\text { El pluralismo } \\
\text { cultural: } \\
\text { preservar y } \\
\text { extender el } \\
\text { pluralismo }\end{array}$ & $\begin{array}{l}\text { Entiende la educación multicultural surge de la no aceptación } \\
\text { por parte de las minorías étnicas de las prácticas de aculturación } \\
\text { y asimilación a las que se encuentran sometidas en el contacto } \\
\text { con las culturas mayoritarias. Para estas minorías ni la } \\
\text { asimilación cultural ni la fusión cultural son aceptables como } \\
\text { objetivos sociales últimos. }\end{array}$ \\
\hline
\end{tabular}




\begin{tabular}{|c|c|c|}
\hline $\begin{array}{l}\text { Brennan \& } \\
\text { Donoghue, } \\
\text { 1974) }\end{array}$ & $\begin{array}{l}\text { La educación } \\
\text { bicultural: la } \\
\text { competencia } \\
\text { en dos culturas }\end{array}$ & $\begin{array}{l}\text { La educación multicultural debería producir sujetos } \\
\text { competentes en dos culturas diferentes. Tal posición es } \\
\text { consecuencia del rechazo por parte de los grupos minoritarios } \\
\text { de la idea de la asimilación. Para estos grupos la cultura nativa } \\
\text { debería mantenerse y preservarse y la cultura dominante } \\
\text { debería adquirirse como una alternativa o segunda cultura. }\end{array}$ \\
\hline $\begin{array}{l}\text { Delgado- } \\
\text { Gaitán } \\
(1992)\end{array}$ & $\begin{array}{l}\text { La educación } \\
\text { como } \\
\text { transformación: } \\
\text { educación } \\
\text { multicultural y } \\
\text { reconstrucción } \\
\text { social }\end{array}$ & $\begin{array}{l}\text { Desde estas posiciones se concibe la educación multicultural } \\
\text { como un proceso encaminado a lograr un desarrollo de los } \\
\text { niveles de conciencia de los estudiantes de minorías, de sus } \\
\text { padres y de la comunidad en general acerca de sus condiciones } \\
\text { socioeconómicas, con objeto de capacitarles para la ejecución } \\
\text { de acciones sociales basadas en una comprensión crítica de la } \\
\text { realidad. }\end{array}$ \\
\hline $\begin{array}{l}\text { (Grinter, } \\
\text { 1992; } \\
\text { Leicester } \\
\text { 1992) }\end{array}$ & $\begin{array}{l}\text { Educación } \\
\text { antirracista }\end{array}$ & $\begin{array}{l}\text { Liberalismo que enfatiza la libertad de pensamiento y acción que } \\
\text { posee cada individuo, sustentador de una educación } \\
\text { multicultural que persigue el entendimiento entre culturas y el } \\
\text { cambio paulatino de la sociedad. Transformación social basada } \\
\text { en la liberación de los grupos oprimidos y la eliminación de las } \\
\text { discriminaciones institucionales, concibiendo la escuela como } \\
\text { una agencia para la promoción de la acción política. }\end{array}$ \\
\hline
\end{tabular}

Fuente: (GARCIA; PULIDO; MONTES, 1997)

Sumado a lo expuesto anteriormente, los programas educativos de formación inicial de profesores en ciencias, deben proponer la decolonialidad del conocimiento como un proceso de resistencia al pensamiento o noción de universalidad del cientificismo y la modernidad que se han instalado abruptamente en la vida de nuestros pueblos. Por lo anterior, vale la pena preguntarse la necesidad de implementar una pedagogía decolonial, vinculada con una reflexión educativa que emancipa una sublevación a las relaciones con las redes de poder que han operado la educación hegemónica, reivindicando así a la escuela como institución que ha direccionado la formación del sujeto moderno por excelencia (DÍAZ, 2010).

En relación a lo anterior, la educación en ciencias debe adicionar una nueva cuestión a su propuesta formativa, educar para construir una sociedad diversa culturalmente; brindando la posibilidad de vivir en contextos heterogéneos, de esta forma construir comunidad desde la multiplicidad de las identidades. Esto puede plantearse como finalidades, extremadamente complejas y ambiciosas; no obstante, es importante remembrar un par de afirmaciones que apuntan a la formación desde la diferencia, la primera, hace alusión a que quizás la escuela no transforme las realidades del contexto, pero sin ella no será posible hacerlo; la 
segunda: hace referencia a que no existe un proyecto de socialización democrático (justicia social, equidad e interculturalidad) más importante que una educación inclusiva, es decir, para todos (ARROYO, 2013).

Con el fin de resumir la idea principal de éste apartado, autores como Molina, Martínez, Mosquera y Mojica (2009) señalan que inmiscuir a la diversidad cultural en la escuela implica tener en cuenta en una primera instancia aquellas acciones abiertas hacia la heterogeneidad y la diferencia, de otra parte, abrir la discusión sobre un enfoque que asuma la condición de lo diverso y lo heterogéneo en relación con las concepciones y cosmovisiones del sujeto.

\section{La diversidad cultural como proyecto social}

Los inicios del siglo XXI, se han caracterizado por una especial celeridad relacionada a la transformación de los sistemas educativos latinoamericanos. Las veloces dinámicas de cambio social, económico, cultural y tecnológico, trazan nuevas exigencias que precisan a los sistemas educativos a una renovación permanente para dar respuesta a las necesidades de las personas y de las sociedades (HIRMAS, 2008).

En la actualidad, la diversidad cultural en la escuela se considera como un tema de altísimo interés y preocupación entre los educadores. La amplia variedad de estudiantes, estilos y ritmos de aprendizaje, diferentes niveles de intereses y motivaciones, ponen en la mesa la necesidad de reconsiderar la escuela tanto en aspectos organizativos como curriculares, requiriendo comprender con urgencia lo que ocurre en el entorno social (RODRÍGUEZ, 2004).

En relación a lo mencionado anteriormente, se debe partir de la comprensión de que la educación en nuestro país subyace de una sociedad diversa, heterogénea desde sus etnias que la componen, clases sociales, géneros, creencias religiosas e incluso cosmopolita al convertirse en centro de interés de otras nacionalidades. En la medida en la que se toma conciencia de que las sociedades son multiculturales

y desiguales, los sistemas educativos no pueden centrarse exclusivamente desde 
perspectivas universales, descontextualizadas de las realidades locales, alejada entonces de una diversidad cultural.

Atendiendo el enorme acervo cultural que destaca nuestra nación y reflexionando sobre esta consideración, la formación docente debe emanciparse como alternativa y respuesta educativa que permita acercar al estudiantado a un conocimiento que representa a una sociedad plural (GUIDO et al., 2013). Entonces, como proyecto social se hace imperativo desde la educación científica romper con la imagen de la ciencia como saber universal y aproximar conocimientos alternos y legítimos de nuestras comunidades a los procesos formativos en nuestras aulas de clases. De otro lado, reivindicar la imagen histórica y social que ha plasmado el conocimiento científico como agente epistemicida y perpetrador de exclusiones culturales, racistas, de género y saberes.

Investigaciones como las de Beltrán (2017), esbozan estos tipos de exclusión en el marco de la globalidad hasta llegar nuestra realidad local (nacional), exponiendo estas situaciones incluso como vejámenes sociales implícitas en nuestros actos de enseñanza de las ciencias naturales, inclusive al plasmarse como recursos de divulgación través de la circulación de revistas especializadas y en textos escolares en Colombia.

Entonces, si lo que instituye el ímpetu del desarrollo se coliga a la capacidad de las sociedades en actuar sobre sí mismas y de transformar el curso de los hechos y de los procesos, la acción global que hoy asume la modernización ante la educación tropieza y tensiona las identidades, conllevando a predisposiciones fundamentalistas que demandan una nueva postura cultural, la cual no debe ser estética, ni dogmática, asumiendo su construcción a través de la alteridad de las colectividades y de los diferentes tipos de conocimientos de circulación científico y no científicos presentes en la escuela.

De acuerdo con lo descrito, la incertidumbre que conlleva las vertiginosas transformaciones sociales en el campo educación en ciencias producto de tendencias foráneas que subyacen desde el marco de lo político y económico (modernidad, globalización), invitan a una profunda reconfiguración de las culturas 
tradicionales (campesinas, indígenas y negras) por la intensificación de su interacción con posicionamientos mayoritarios y hegemónicos, percibiéndose como una amenaza a la supervivencia de las mismas Mato (2005). De tal forma, Walsh (2012) señala la importancia de una reflexión pedagógica que dé lugar desde la escuela como proyecto social que apunte a liberación de movimientos conceptuales universales proponiendo prácticas de resistencia como estrategias pedagógicas para transgredir la imposición colonial.

Finalmente, la respuesta a este proyecto radica en la coexistencia cultural a través del dialogo y de la interacción, es decir, comprender la importancia del conocimiento científico en el desarrollo de la humanidad sin desconocer las realidades locales y sus saberes propios, ubicando a través de un diálogo intercultural el conjunto de significados y cosmovisiones que se encuentran en la escuela, esta apuesta semiótica incluye las relaciones culturales del contexto local y regional, con el fin de revitalizar identidades étnicas, promover sinergias en la interacción de todos los agentes educativos y sociales, democratizar el saber y establecer espacios de consensos y negociación, para la construcción del conocimiento escolar (URIBE, 2019).

La diversidad cultural como proyecto político

Para comprender la situación actual que ciñe las políticas que atienden a la diversidad cultural, la UNESCO (2005) propone la siguiente reflexión:

La globalización se nos presenta como una oportunidad de intercambio y enriquecimiento entre naciones y personas, pero también introduce nuevas tensiones en la convivencia social. Advertimos el surgimiento de nuevas formas de intolerancia y agresión. Por un lado, experimentamos la fascinante proximidad de múltiples culturas; pero por otro vemos cómo aumentan la xenofobia, el racismo y las discriminaciones, en diferencias de color, sexo o rasgos étnicos. La diversidad cultural, en lugar de ser considerada como patrimonio común de la humanidad y oportunidad de crecimiento, se convierte en amenaza, y es utilizada como 
excusa para la intolerancia y la discriminación (UNESCO, 2005, p.8).

La diversidad cultural es un distintivo esencial de la humanidad y un componente clave de su desarrollo. Colombia es un país reconocido por su compleja e inmensurable diversidad cultural que se expresa en una gran pluralidad de identidades y de expresiones culturales de los pueblos y comunidades que forman la nación (MINISTERIO DE CULTURA, 2009).

La política pública de diversidad cultural subyace de la Constitución Política de 1991, la legislación cultural nacional y las normas derivadas de ellas, como a su vez en atención a normas internacionales tales como el Convenio 169 de la OIT (Organización Internacional del Trabajo).

La política de diversidad cultural busca contribuir a la adopción de un régimen legal de protección de las expresiones y manifestaciones culturales indígenas y afrocolombianos como propiedad colectiva de los pueblos y a la salvaguardia del saber tradicional. En relación a la primera disposición el Ministerio de Cultura reglamentó la Ley General de cultural 1185 de 2008 y el Decreto 2941 de 2009, estableciendo los mecanismos más adecuados para la protección del acervo cultural entre ellos la riqueza indígena. De otra parte, se establecen los Decretos $4181 / 2007$ y $4401 / 2008$ que avalan la comisión intersectorial para el avance de la población afrocolombiana, palenquera y raizal en el país.

Dentro de las políticas educativas que abordan la diversidad cultural, la Ley 115 General de Educación desde las disposiciones preliminares en el Artículo 5ํㅜ Numeral 6 plantea "el estudio y la comprensión crítica de la cultura nacional y de la diversidad étnica y cultural del país, como fundamento de la unidad nacional y de su identidad". A estas políticas se suma el Decreto 1122 de junio 18 de 1998, la cual expide las normas para el desarrollo de la Cátedra de Estudios Afrocolombianos en todos los establecimientos de educación formal del país:

Es de resaltar que la Ley 115 de 1994, en su sección tercera educación básica en el Artículo 23ำ establece como área obligatoria y fundamental a la asignatura en Ciencias Naturales y Educación Ambiental, la cual deberá atender a 
lo estipulado en los artículos 13, 45, 51, 56, 57, 76, 77, 104, 142, 144 у 159, relacionados al respeto y apropiación a la diversidad cultural de nuestra nación. En correspondencia a la creación y establecimiento de Programas de Formación Inicial de Profesores (Licenciaturas), a través de la Ley 1188/2008, el Decreto 1295/2010, el Decreto 1075/2015 y el Decreto 1330/2019, se expide la reglamentación del sector educación y se establecen las pautas para obtener el registro calificado de los programas académicos de educación superior, esto permitirá la oferta y desarrollo de los mismos, a lo que se debe sumar la atención a los lineamientos de calidad propuestos por el Ministerio de Educación Nacional (2014), en donde estas directrices desde su discurso político invitan a la realización de una propuesta formativa que propenda por la incorporación de la diversidad cultural como eje fundante del perfil y competencias del futuro egresado, esperando que esto redunde desde su profesión en el desarrollo y apropiación local, respeto y valoración del territorio.

En relación a lo anterior, con la intensión de superar las brechas descritas, Hirmas (2008) hace una propuesta muy interesante que permite reflexionar sobre tres aspectos a tener en cuenta (pertinencia, convivencia e inclusión) para gestar un proyecto político educativo que apunte a la diversidad cultural:

En primer lugar, la pertinencia reflexiona sobre la relevancia cultural y significación de los aprendizajes que se dan en la escuela, en ese sentido, si se delibera sobre los afectos que consolidan la identidad, tal como reconocer las experiencias de los estudiantes, al igual que los saberes previos y cosmovisiones sobre el mundo (atención a las realidades al ingresar a la escuela). Una educación asertiva e incluyente considera la procedencia social y cultural de cada persona, así como la individualidad de sus características.

El segundo aspecto se relaciona con el estudio de los diversos tipos de convivencia, en torno a las actitudes, valores y comportamientos, de aprobación o rechazo, de entendimiento o confrontación, de inclusión o exclusión, de alteridad o intolerancia. Lo que verdaderamente enaltece el desarrollo de las sociedades y de las personas es la relación y el diálogo con las diferencias. La apreciación y la 
experiencia de la diversidad permiten al ser humano revelar, construir y reafirmar su propia identidad al distinguirse de otros.

La tercera característica hace referencia a la inclusión. En otros términos, si la escuela favorece una educación incluyente y atiende a sus estudiantes minimizando la desigualdad de oportunidades con que ingresan y permanecen en ella, cualquiera sea situación cultural o social. La educación diversa culturalmente, además de responder a la diferencia en términos de la congruencia de los aprendizajes y de la formación humana pluralista, establece canales o redes de enseñanza y aprendizaje de las ciencias, favoreciendo el dialogo entre saberes propios o ancestrales con conocimientos científicos o universales, tal como lo afirmar Uribe (2019), esto implica conectar diversas epistemologías con el fin de generar un diálogo interactivo, deliberativo y dialógico en el aula de clase, contribuyendo a consolidar puentes entre conocimientos, reconociendo el contexto, los territorios y las comunidades.

\section{LA DIVERSIDAD CULTURAL COMO ESTRATEGIA TRANSVERSAL DEL CURRICULO DE PROGRAMAS DE FORMACIÓN INICIAL DE PROFESORES DE CIENCIAS NATURALES Y EDUCACIÓN AMBIENTAL}

Pensamientos globalizados, competitividad, estandarización y universalidad del conocimiento, delinean en la actualidad las sendas de la educación, esto lleva a reflexionar sobre la función que recae sobre la escuela en torno a la formación del sujeto desde otra realidad, la de un contexto sociocultural heterogéneo, entonces, parafraseando a Lluch (2011) desde algunos de sus cuestionamientos sobre la educación intercultural surgiría como pregunta: ¿cómo educar desde la diversidad en sociedades cohesionadas?

Dicha consideración, lleva a reflexionar sobre el papel que cumple el currículo escolar como una estrategia que confiere a docentes y estudiantes la posibilidad de construcción de identidad a partir de la diferencia, esto, nos invita a ir más allá de la mera organización de contenidos temáticos y programáticos de los planes de 
estudio, nos convida a comprender las necesidades de nuestro contexto conforme a las realidades formativas del mismo (MORA, 2009).

A su vez, los proyectos curriculares posicionados desde la diversidad cultural suponen promover programas para el conocimiento de aquellas culturas minoritarias cada vez más presentes en nuestros centros educativos, evitando así el asimilacionismo frente a la cultura hegemónica dominante, esto en pos de favorecer el desarrollo de las relaciones interpersonales en un plano de equidad (NÚÑEZ, 2009). Lluch (2011) realiza un diagnóstico con el fin de conocer aquellas experiencias que subyacen de enfoques curriculares que reconocen contextos multiculturalidad:

Tabla 1. Enfoques de currículos de la educación Multicultural

\begin{tabular}{|c|c|c|}
\hline $\begin{array}{c}\text { AUTORE } \\
\mathrm{S}\end{array}$ & ENFOQUE & ESTRATEGIA \\
\hline \multirow{3}{*}{$\begin{array}{l}\text { McCarthy } \\
\text { (1994) }\end{array}$} & Socialdemócratas & $\begin{array}{l}\text { Estas propuestas curriculares subrayaban la importancia de } \\
\text { los programas de educación bilingüe y planteaban la } \\
\text { necesidad de realizar "añadidos" curriculares que supusieran } \\
\text { el conocimiento de la lengua y cultura de esos grupos } \\
\text { culturales. }\end{array}$ \\
\hline & Conservador & $\begin{array}{l}\text { Esta perspectiva entiende que la consideración de la } \\
\text { pluralidad y la diversidad cultural en el currículum supone una } \\
\text { amenaza. Apuesta por la conformación de currículum donde } \\
\text { la representatividad cultural sea inequívoca con objeto de } \\
\text { evitar lo que a su juicio supondría una inevitable disgregación } \\
\text { cultural. }\end{array}$ \\
\hline & Críticos & $\begin{array}{l}\text { Este tercer enfoque o manera de entender la educación } \\
\text { multicultural surge de la no aceptación por parte de las } \\
\text { minorías étnicas de las prácticas de aculturación y asimilación } \\
\text { a las que se encuentran sometidas en el contacto con las } \\
\text { culturas mayoritarias. }\end{array}$ \\
\hline $\begin{array}{l}\text { (Morrill, } \\
\text { 1987; } \\
\text { Burger, } \\
\text { 1969; } \\
\text { Brennan \& } \\
\text { Donoghue } \\
\quad \text {,1974) }\end{array}$ & $\begin{array}{l}\text { La educación } \\
\text { bicultural: la } \\
\text { competencia en } \\
\text { dos culturas }\end{array}$ & $\begin{array}{l}\text { La educación multicultural debería producir sujetos } \\
\text { competentes en dos culturas diferentes. Tal posición es } \\
\text { consecuencia del rechazo por parte de los grupos minoritarios } \\
\text { de la idea de la asimilación desarrollo de competencias } \\
\text { culturales, pues se entiende como un elemento decisivo en la } \\
\text { labor de "puente" entre dos culturas. }\end{array}$ \\
\hline $\begin{array}{l}\text { Lovelace } \\
\text { (1995) }\end{array}$ & $\begin{array}{l}\text { Perspectiva de las } \\
\text { contribuciones }\end{array}$ & $\begin{array}{l}\text { Consiste en la incorporación de contenidos referidos a las } \\
\text { características culturales de distintas minorías del centro } \\
\text { escolar. Los rasgos que se explican se centran en aspectos } \\
\text { simbólicos: artesanía, música, gastronomía, personajes }\end{array}$ \\
\hline
\end{tabular}




\begin{tabular}{|l|l|l|}
\hline \multirow{2}{*}{\begin{tabular}{|l|l|} 
Perspectiva aditiva. & $\begin{array}{l}\text { célebres, fechas importantes de su calendario cultural, } \\
\text { celebraciones. }\end{array}$ \\
\cline { 2 - 3 } & $\begin{array}{l}\text { Se plantea añadir unidades didácticas, temas, trabajo al } \\
\text { currículum ordinario. De esta forma, tampoco hay } \\
\text { modificación de la propuesta curricular prescriptiva pero sí se } \\
\text { plantea la necesidad de completar el currículum para tratar la } \\
\text { diversidad cultural. }\end{array}$ \\
\hline transformación del \\
currículum & $\begin{array}{l}\text { Este enfoque se diferencia de los anteriores planteando, de } \\
\text { entrada, un cambio curricular para todos los alumnos y } \\
\text { alumnas. El objetivo es permitir a todo el alumnado la } \\
\text { consideración de los contenidos curriculares desde diferentes } \\
\text { perspectivas culturales. }\end{array}$ \\
\hline Perspectiva de \\
acción cívica.
\end{tabular}} & $\begin{array}{l}\text { Esta perspectiva implica, profundizar en el enfoque anterior. } \\
\text { Se trata de educar al alumnado para que, además de la } \\
\text { comprensión cognitiva y el desarrollo de determinadas } \\
\text { actitudes, comprometa su acción y traslade estas actitudes a } \\
\text { una conducta responsable ante situaciones de injusticia, } \\
\text { discriminación, marginación. }\end{array}$ \\
\hline
\end{tabular}
Fuente: LLUCH (2011)

La formulación de un currículo que opte por la diversidad cultural como eje transversal, tiene como propósito prescindir de la introducción de modelos educativos externos, para intentar construir una pedagogía propia, sustentada en un modelo acorde a las necesidades educativas de nuestro contexto, planteando nuevos referentes teóricos y metodológicos para su aplicación en donde siempre se propenda por un diálogo intercultural (URIBE, 2019).

Entonces, una educación orientada desde la diversidad cultural debe perseguir objetivos en la enseñanza de las ciencias como: a) una alternativa que potencie los discursos en el aula a través de un dialogo intercultural entre los saberes propios y el conocimiento científico como estrategia de enseñanza y aprendizaje contextualizada; b) Propender como fundamento del conocimiento escolar por el desarrollo de valores tales como la alteridad, correspondencia, cooperación, inclusión y respeto por la diferencia, y c) apropiar y conservar el acervo cultural de una nación tan diversa como lo es Colombia.

En relación a todo lo expuesto, una estrategia que conduzca a un currículo orientado desde la diversidad cultural como eje transversal para los PFILCNYEA deberá reflexionar sobre dos aspectos que son imprescindibles y delineantes del 
mismo, como lo son los contenidos curriculares, además de las fuentes y criterios de selección de dichos contenidos.

Los Contenidos Curriculares: corresponden a los tipos de contenidos que se encuentran implícitos en los planes de estudio desde el macro, meso y microcurrículo, siendo un punto de referencia del objeto de la práctica pedagógica mediada en el proceso de enseñanza y aprendizaje de las ciencias naturales.

En este sentido sobresalen aspectos tales como conceptos, procedimientos, actitudes y valores. Los conceptos hacen referencia a aquellas concepciones presentes en los sujetos sobre saberes propios, saberes ancestrales y saberes tradicionales que dialogan desde un mismo nivel de significancia frente conocimientos de la ciencia occidental. Los procedimientos se relacionan con las prácticas educativas, las cuales de gran manera se ciñen al syllabus (microcurrículo) de las asignaturas como al diseño y aplicación de sus unidades didácticas, las que deben ser pensadas y orientadas desde la diversidad cultural. Así mismo, se hace imperativo consolidar todo un campo de pensamiento para lo cual es necesario la reflexión e innovación a través de procesos desarrollados por medio líneas de investigación sobre el tema. Las actitudes deben hacerse visibles a través del vincular a la diversidad cultural como centro de interés formativo, como a su vez la diversidad ecosistémica y de los recursos naturales, lo que redundará sin duda alguna en la apropiación del territorio y la nación. Finalmente, desde la construcción curricular se hace necesario plasmar algunos valores tales como la Inclusión, reciprocidad, tolerancia, cooperación y respeto a la diferencia, con el fin de que los sujetos desde su preparación reconozcan la heterogeneidad del conocimiento, la sociedad y la cultura, rompiendo la estructura hegemónica de formación universalista provista desde la modernidad y la globalización.

De otra parte, en relación a las fuentes y criterios de selección de contenidos curriculares, estas aluden al fundamento que utiliza los PFILCNYEA para seleccionar los contenidos a impartir (esto aplica también para los docentes), tales como experiencias, textos escolares y políticas educativas. La experiencia hace referencia al contexto en donde se desenvuelve el acto educativo, de tal forma, se 
hace imprescindible reconocer las realidades presentes para el sujeto (experiencia personal) o los sujetos (experiencia de grupo) de acuerdo a sus subjetividades y la significancia de las mismas posicionadas bajo el marco de la diversidad cultural. De esta misma manera, los textos escolares corresponden a libros, revistas especializadas, artículos, bases de datos, repositorios u otro tipo de material de circulación física o digital que aborda temáticas y/o contenidos programáticos de las asignaturas, cabe mencionar que al realizar la selección de los mismas, estos deben contextualizar la ciencia local con la universal, de la misma manera de presentar una ciencia la cual no sea racista, discriminatoria frente al género, la diversidad socio-económica o la pluralidad epistémica. Por último, la gestación del currículo, debe atender a las políticas nacionales o internacionales que vinculen aspectos relacionados a la cultura o su diversidad, de la misma manera, presentar una postura reflexiva y critica frente a lineamientos o directrices que promuevan la homogenización y/o estandarización de la misma.

Así pues, un currículum orientado desde la diversidad cultural convendrá a la comprensión y conceptualización de la realidad social desde los más variados tamices culturales; buscando permearle de forma transversal en su totalidad. En definitiva, esta apuesta pretende desarrollar competencias en el estudiantado y profesorado, que posibiliten entender el mundo desde diversas lecturas culturales, reflexionando desde la alteridad y la diferencia sobre las mismas, favoreciendo una postura y actitud asertiva, comprometida, enriquecedora y contextualizada, de alta significancia para el proceso de enseñanza y aprendizaje de las ciencias naturales (MORA, 2009; NÚÑEZ, 2009; LLUCH, 2005).

En este sentido, comprender un currículo que se enmarque en la educación para la diversidad, subyace en entender la heterogeneidad presente en el aula, haciéndose necesario pensar y diseñar la forma de trabajar para una educación superior incluyente. Esto, conlleva no solamente a discrepar de modelos organizadores y didácticos de la escuela tradicional homogeneizadora, sino a reflexionar en relación a una evaluación de la heterogeneidad de los aprendizajes. 
En relación a lo anterior, la universidad se convierte en un escenario de formación en donde se ponen en manifiesto no solamente diferentes formas de enseñanza sino a su vez diversos tipos de aprendizajes, buscando una aproximación sobre aquello que se desea enseñar y lo que se aprende; lo cual se presenta como espacio propicio de conocimientos y saberes compartidos generadores de pensamientos.

Entonces, pensar en construir una propuesta curricular para la formación de educadores en ciencias, ha de entender que el estudiante deber ser protagonista de su proceso de aprendizaje, lo cual no significa que sea el único responsable del mismo; de tal forma, la enseñanza y evaluación debe centrarse a partir de metodologías activas, las cuales potencien el desarrollo de escenarios de aprendizaje situados, conectando con casos y situaciones lo más reales posibles al mundo laboral, social y cultural (SÁNCHEZ, 2007); por consiguiente , la evaluación se convierte en una herramienta esencial para emitir juicios justos y objetivos sobre el proceso de aprendizaje del docente en formación, producto de actividades significativas y contextualizadas.

Con el fin de descolonizar el conocimiento, y propiciar un dialogo intercultural que vincule diferentes tipos de saberes presentes en los diversos miembros de la comunidad educativa, valdría la pena reflexionar sobre la evaluación de aprendizajes desde un enfoque de diversidad cultural; éste, deberá fundamentarse en las singularidades del grupo de estudiantes, atendiendo las características culturales, sociales, económicas y personales de los mismos, favoreciendo la cohesión del grupo. La valoración debe promover una retroalimentación del conocimiento hacia el respeto y la singularidad, pues han de tenerse en cuenta las capacidades, habilidades, intereses, estilos de aprendizaje y respuesta del estudiantado apreciando la diferencia de forma equitativa; a estos se suman resultados de experiencias de trabajo cooperativo que promuevan oportunidades de participación y motivación, que conlleven a la integración de los sujetos (SÁNCHEZ, 2007).

En relación a lo anterior, Collicot (2000) hace referencia en como pensar una evaluación integral e incluyente, aludiendo la importancia de planificar la enseñanza 
desde la flexibilización del conocimiento, visualizando la heterogeneidad de estudiantes que confluyen en una misma lección, reduciendo así, la necesidad de impartir programas paralelos al propiciar el dialogo entre los saberes presente en dicho espacio, en este sentido, la formación inicial de profesores se encuentra ante un desafío, una cualificación desde la diversidad.

Finalmente, una evaluación posicionada desde la diversidad cultural debe conllevar al futuro licenciado en ciencias a: considerar diversos tipos de aprendizaje, reconocer la alteridad y otredad presente en el estudiantado, planificar cómo se va a presentar la información, propiciar una educación inclusiva, reconocer la diversidad epistémica presente en el aula de clases e incentivar el respeto por la diferencia.

\section{LA CIENCIA VISTA DESDE LA DIVERSIDAD CULTURAL: PERSPECTIVAS DE UN CURRICULO DE FORMACIÓN INICIAL DE PROFESORES}

La descolonización del conocimiento a partir de la investigación educativa en ciencias naturales y educación ambiental

Permanentemente la educación ha sido afectada por el entorno social que le rodea. Dentro de los procesos de transformación y mejora vinculados al desarrollo personal y de la sociedad, se hace imprescindible tener como referente el contexto en el que se enmarca. Los cambios sociales, culturales y económicos intervienen de forma determinante en la planeación educativa, exigiendo de tal manera, modificaciones en la consolidación de los sistemas educativos y que, en relación a la formación docente, requiere de investigación y reflexión para la transformación de su práctica (TEJADA, 2001).

Entonces, la educación se concibe como un vehículo que favorece a la construcción y el desarrollo de capacidades para actuar y participar en sociedad. De ello se desprende que la educación es funcional, ya que posibilita intervenir en la dinámica de la sociedad, resolver conflictos y participar en su construcción (RAEL, 2009). 
La consolidación de la identidad del maestro, se enmarca por componentes determinantes, como lo son: creencias, concepciones, actitudes, percepciones, pensamientos, sentimientos, emociones, etc., que bien pueden sintetizar su estructura personal y socio-didáctica (DOMÍNGUEZ, 2006); por tal razón, el docente desde su formación debe aprender a conocerse a sí mismo, para así reconocer y respetar las diferencias que puede encontrar en el desarrollo de su profesión en relación con los demás agentes sociales. Comprender que existe diversidad cultural y social, conlleva a la generación de una nueva perspectiva que permite valorar estos aspectos y trabajar por un sistema educativo más incluyente (OLMO; HERNÁNDEZ, 2004).

En relación con lo anterior, el docente de ciencias en formación debe reconocer la diversidad cultural, no solo como una característica de la sociedad que le permite apreciar y comprender las diferencias entre individuos, sino como una oportunidad de enriquecer su desarrollo conceptual y discurso educativo (LE METAIS, 2002).

La necesidad de aportar ideas, concepciones y prácticas que favorezcan la formación del profesorado, se puede llevar a cabo a través de la aproximación de estrategias, modelos y herramientas que en la actualidad se desarrollan desde la investigación e innovación de la educación en ciencias (ADÚRIZ-BRAVO; IZQUIERDO, 2002), que en este aspecto, aproximen a los docentes en formación a un pensamiento abierto desde la diversidad cultural y social, que a partir de la reflexión y el autodesarrollo propicie a la consolidación de su identidad profesional (DOMÍNGUEZ, 2006).

Todo lo descrito anteriormente, conlleva a reflexionar sobre ¿cuál ha de ser entonces el posicionamiento de los futuros licenciados en ciencia, en relación a la concepción del conocimiento ante un país pluriétnico y multicultural como lo es Colombia? Tal vez, la respuesta a este cuestionamiento puede darse a partir del diseño y aplicación de proyectos de investigación que convoquen a reflexionar sobre la deshomogenización del conocimiento y a la reivindicación de los saberes tradicionales y populares en la escuela. 
Tal como es puesto en manifiesto por Uribe (2019), los programas de formación de profesores están llamados a trabajar con y por las comunidades indígenas, afrodescendientes, campesinas e inmigrantes del país, para conocer sus realidades, su historia, sus saberes y tradiciones, buscado relacionar todo lo anterior al acto educativo. Tomando como referente a Walsh (2009) la investigación educativa desde el marco de la diversidad cultural y la interculturalidad, surge como alternativa para promover un pensamiento crítico y emancipatorio que permita superar el colonialismo conceptual arraigado a estructuras políticas, sociales y de poder, existentes desde hace siglos en el ámbito escolar latinoamericano. Sin duda alguna, la investigación en esta área genera nuevos planteamientos desde campos de pensamiento teórico-metodológicos en la educación superior, develando sentidos de diversidad cultural en la construcción y consolidación de la identidad de futuros docentes.

\section{HACIA LA CONSOLIDACIÓN DE UN MODELO EDUCATIVO POSICIONADO DESDE LA DIVERSIDAD CULTURAL}

En la actualidad, se proyecta al maestro como un actor reflexivo del contexto en el cual se desenvuelve, competente en su campo de acción desde la gestión, planificación y evaluación. Agente proactivo que desde la investigación e innovación sea capaz de aportar conocimientos, que permitan abordar y dar solución a las demandas socio-educativas relacionadas desde la diversidad de los aprendizajes en su práctica educativa (ERICE, 2004). La formación del profesorado implica según eso, una auténtica y completa renovación conceptual, metodológica y actitudinal, que confluye en la construcción de un nuevo tipo de docente para una nueva educación (GONZÁLEZ, 1998), que para el contexto de nuestra nación, evoca una reivindicación de nuestro acervo cultural, al respeto y exaltación de nuestros saberes populares y ancestrales a través de un dialogo con los conocimientos occidentales, llevando a pensar un nuevo modelo educativo, un modelo propio que consolide una pedagogía y didáctica instaurada desde el campo de nuestra diversidad cultural. 
Como alternativa a lo descrito anteriormente, la educación popular de Freire constituye una corriente de pensamiento, cuyo horizonte emancipador emplea propuestas metodológicas, pedagógicas y didácticas basadas en la participación y el diálogo, buscando la reivindicando de saberes como alternativa ante modelos hegemónicos, en pos del detrimento de las injusticias que han hecho parte del proceso formativo a través de su práctica (GUELMAN, 2018). Entonces, un programa de formación inicial de profesores posicionado desde un modelo educativo popular, debe rescatar los conocimientos previos del sujeto, así como sus tradiciones populares y culturales más diversas, permitiendo legitimar desde el proceso formativo su identidad.

De otra parte, en búsqueda de la instauración de un modelo educativo contextualizado, se hace necesario reconocer la importancia de tendencias actuales que marcan el rumbo de la educación actual, eso sí, sin permitir la transgresión o imposición de las mismas sobre lo propio; de tal forma, la educación intercultural hace sinergia sobre el conjunto de significados y cosmovisiones presentes en el ámbito educativo, para el diseño de propuestas de enseñanza y aprendizaje desde la diversidad cultural, lo que permite incluir considerados culturales del contexto local y regional, posibilitando reestablecer identidades étnicas, promoviendo asociaciones y redes de interacción de todos los agentes educativos y sociales, democratizando el saber y estableciendo espacios de consensos y negociación, para la construcción conjunta de un currículo basado en el desarrollo de capacidades interculturales (URIBE, 2019).

\section{DEL UNIVERSALISMO AL CONOCIMIENTO DIVERSO: RELACIONES ENTRE FORMACIÓN DEL PROFESORADO EN CIENCIAS, CAMBIO DIDÁCTICO Y PRÁCTICAS EDUCATIVAS}

En relación a la formación inicial del profesor de ciencias, Mellado (1996) hace referencia que en dicho proceso se evidencian algunos conocimientos los cuales denomina estáticos y dinámicos, en donde los primeros incluye aquellos conocimientos académicos que pueden ser independientes de la persona concreta 
que enseña y del contexto específico donde se desarrolla la actividad docente, tales como los conocimientos propios de la ciencia, conocimientos psicopedagógicos generales y los conocimientos teóricos de la didáctica de las ciencias; de otra parte, el componente dinámico se genera y evoluciona a partir de los propios conocimientos, creencias y actitudes del estudiante, pero requiere de reflexión personal y de la práctica de la enseñanza de la materia específica en contextos escolares concretos. En relación a lo anterior, Lederman (1992) hace referencia que las prácticas pedagógicas en gran medida (incluso para los docentes en formación), parecen depender más de su conocimiento didáctico del contenido (CDC), es decir, un conocimiento que se hace más fuerte con respecto a la experticia que crece en torno a la práctica educativa; no obstante, Mellado (1996) hace hincapié que dicha actividad se encuentra permeada por lo general a partir de una serie tradiciones y creencias a menudo implícitas sobre la forma de enseñar.

En tal sentido, se hace imprescindible reconocer la epistemología docente, esto vislumbra la senda del entendimiento y comprensión de los conocimientos y las actitudes de los profesores en torno a la enseñanza, el aprendizaje, la evaluación y en definitiva el currículo, lo que en términos generales significa conocer las características de la práctica docente. Dicho de otra forma, no tener en cuenta la epistemología del docente al momento de diseñar programas de formación inicial o continuada del profesorado de ciencias, puede constituirse en un serio impedimento para la estructuración de los mismos (MOSQUERA, 2008).

Colombia enmarca una fuerte tendencia hacia el universalismo del conocimiento, en donde la ciencia occidental se sobrepone sobre otros tipos de saberes, expresándose de esta manera una epistemología positivista en los docentes de los programas de formación inicial de profesores de ciencias naturales y educación ambiental en Colombia. Producto de esto, hace necesario reflexionar sobre ¿cuál será la incidencia de enseñar una ciencia occidental en un país diverso epistémicamente como el nuestro?

Lo anterior, se presenta como un cuestionamiento ante una respuesta evidente, la violencia epistémica. Históricamente en nuestros pueblos latino- 
americanos se ha impuesto un conocimiento hegemónico o universal (WALSH, 2009), conocimiento que ha llevado al detrimento de nuestro acervo cultural y la perdida de nuestra identidad, un conocimiento impuesto por políticas y tendencias económicas que poco a poco se ha encargado de enculturalizarnos, en otras palabras, un colonialismo desde la modernidad. Cabe resaltar que el cientificismo o universalismo, subyace como una ruta para acceder al conocimiento, en donde vale la pena mencionar que no es la única, esto convoca desde el acto formativo no solo a reconocer un pluralismo epistémico, sino a interponer un dialogo entre los diferentes conocimientos, dando mayor significado a los mismos. Tal como manifiesta Uribe (2019), en América el producto de la lucha y resistencia de los pueblos indígenas ha gestado universidades de carácter intercultural, siendo un esfuerzo histórico de descolonizar la educación y el sistema educativo en todos los niveles de enseñanza incluyendo la educación superior. Dichas instituciones buscan promover un diálogo intercultural, entre la cosmovisión indígena y la visión de modernidad, esperando romper la tradición occidental que ha justificado el marco del cual subyace la universidad, logrando reivindicar de los pueblos originarios y a poner en relación la diversidad de epistemes, partiendo de lo local y regional.

Mosquera (2008) expresa las dificultades que se plantean en la transformación del currículo, estas pueden interesar a los profesores si se favorecen reflexiones conscientes, críticas y analíticas acerca de la necesidad de construir nuevos cuerpos teóricos que ayuden a interpretar de otra manera la actividad científica, la naturaleza de la ciencia y la metodología de investigación científica, que para este caso, se complementa y consolida a través de un dialogo de saberes a partir de la interacción con diversidad de conocimientos propios, ancestrales y tradicionales; entonces, una transformación curricular en la formación de docentes, puede conllevar a la reflexión epistemológica que instaure una nueva línea de pensamiento, la cual se espera pueda redundar en futuras prácticas educativas más próximas a la demanda del contexto y del territorio, esperando incurra en un cambio didáctico positivo sobre las nuevas generaciones en torno a concepciones diversas culturalmente sobre la ciencia. 


\section{EPÍLOGO}

Perspectivas como la epistemología del sur, subyacen como alternativas que permite reivindicar los saberes propios de nuestra nación, frente aquellos conocimientos universales agentes del progreso y de la modernidad, que desde la occidentalización del mundo no son nada menos que instrumentos de homogenización de la sociedad. En relación a lo expuesto, surgen como propuestas la descolonización del conocimiento dentro de la formación docente, permitiendo reconocer a la diversidad cultural como una riqueza que potencia el acto educativo dentro de los procesos de enseñanza y aprendizaje de las ciencias naturales, favoreciendo la conservación de nuestro acervo y estableciendo rutas que conduzcan a un dialogo intercultural en la escuela, por medio de una apertura de las concepciones de los sujetos a través de la comprensión e interacción de pluralidades epistémicas. De otra parte, debe entenderse la diversidad cultural como un proyecto educativo, social y político, esperando que dichos aspectos puedan fundamentar la configuración de los Programas de Formación Inicial de Profesores de Ciencias Naturales y Educación Ambiental, lo que se espera redunde en una potenciación de discursos académicos, los cuales trasciendan pragmáticamente a la conformación de una sociedad más humana que propenda por la alteridad, correspondencia, cooperación, inclusión y respeto por la diferencia entre individuos, como a su vez apropiación por los distintos tipos de diversidad presentes en nuestro territorio. Finalmente, se exponen diversos enfoques curriculares que han buscado la implementación de un pensamiento multicultural en la escuela, desde allí surge como consideración una estrategia para desarrollar un currículo instituido desde la diversidad cultural en PFILCNYEA, que desde las fuentes y criterios de selección (experiencias, textos escolares y políticas educativas) se estructurarán los contenidos curriculares que fundamentan los conceptos, procedimientos y actitudes de los docentes en formación, teniendo como propósito relegar la introducción de modelos educativos externos, de esta manera construir una identidad educativa propia, sustentada bajo nuestros referentes 
teóricos y metodológicos ajustados a las necesidades de nuestro contexto, propendiendo por un dialogo entre saberes populares con los científicos, en donde estos discursos presentan iguales niveles de significancia.

\section{REFERENCIAS}

ADAME, Juan David. Concepciones de ciencia desde la perspectiva de la diversidad cultural en docentes de programas de Licenciatua en Ciencias Naturales y Educación Ambiental en Colombia. Tésis (Doctorado Interinstitucional en Educación con Énfasis en Educación en Ciencias) Universidad Distrital Francisco José de Caldas, Bogotá, 2020.

ADÚRIZ-BRAVO, Agustín.; IZQUIERDO, Merced. Acerca de la didáctica de las ciencias como disciplina autónoma. Revista Electrónica de la Enseñanza de las Ciencias, v 1, p. 180-188, 2002.

ARROYO, María José. La educación intercultural un camino hacia la inclusión educativa. Revista de Educación Inclusiva, v. 6, n. 2. p. 144-159, 2013.

BELTRÁN, María Juliana. Racismo científico y textos escolares de Ciencias naturales (1979-2015). Voces y Silencios: Revista Latinoamericana de Educación, v. 8, n. 1, p. 37-59, 2017.

COLLICOT, Jean. Posar en pràctica l'ensenyament multinivell: estratègies per als mestres. Suports: Revista Catalana d'Educació Especial i Atenció a la Diversitat, v. 4, n. 1. pp. 87-99, 2000.

SOUSA SANTOS, Boaventura. Epistemologías del Sur: utopía y praxis latinoamericana, v. 54, p. 17-39, 2011.

DÍAZ, Cristhian James. Hacia una pedagogía en clave decolonial: entre aperturas, búsquedas y posibilidades. Tabula Rasa, v. 13, p. 217-233, 2010.

DOMíNGUEZ, María. Formación del profesorado de Ciencias Sociales en Educación. Enseñanza de las Ciencias Sociales, p. 1-15, 2006.

DUSSEL, Enrique. 1492: El encubrimiento del Otro: hacia el origen del "Mito de la Modernidad". La Paz: Plural Editores, 1994. 
ERICE, Nélida. Escuela, discapacidad y resiliencia. In: ERICE, Nélida. Alternativas de la diversidad social: las personas con discapacidad. Buenos Aires: Siglo XXI, 2004.

GARCÍA, Francisco; PULIDO, Rafael; MONTES, Ángel. La educación multicultural y el concepto de cultura. Revista Iberoamericana de Educación, v.13, p. 223-256, 1997.

GÓMEZ, Esperanza., PATIÑO, Marisol., BARRETO, Esterla., GONZÁLEZ, Francisco., RIVERA, Jessenia., MUÑOZ John, ROMÁN, Martín. Diversidades y decolonialidad del saber en las Ciencias Sociales y el Trabajo Social. Medellín: Pulso \& Letra Editores, 2014.

GONZÁLEZ, María. La micropolítica de las organizaciones escolares. Revista de Educación, n. 316, pp. 215-240, 1998.

GUELMAN, Anahí; CABALUZ, Fabián; SALAZAR, Mónica. Educación popular y pedagogías críticas en América Latina y el Caribe. In: SALAZAR, Mónica. Corrientes emancipatorias para la educación pública del siglo XXI. Buenos Aires: CLACSO, 2018.

GUIDO, Sandra., GARCÍA, Diana., LARA, Gabriel., JUTINICO, María., BENAVIDEZ, Angie., DELGADILLO, Ingrid., BONILLA Holman. Experiencias de educación indígena en Colombia: entre prácticas pedagógicas y políticas para la educación de grupos étnicos. Bogotá: Universidad Pedagógica Nacional CIUP, 2013.

HIRMAS, Carolina. Educación y diversidad cultural: lecciones desde la práctica innovadora en América Latina. Santiago: OR Caribe, 2008.

LANDER, Edgardo. Ciencias sociales: saberes coloniales y eurocéntrico. Buenos Aires: CLACSO, 2000.

LE MÉTAIS, Joanna. Síntesis Introductoria. In: LE MÉTAIS, J. Hacia una Europa diferente: Respuestas educativas en la interculturalidad. Madrid: CIDE-CIDROE, 2002.

LLUCH, Xavier. Aula intercultural. El portal de la educación intercultural. Dirección General de Inclusión y Atención Humanitaria del Ministerio de Inclusión, Productor, \& FeSP UGT. 2011.

MATO, Daniel. Cultura, política y sociedad. Perspectivas latinoamericanas. Buenos Aires: CLACSO, Consejo Latinoamericano de Ciencias Sociales. 2005. Disponible 
en <http://bibliotecavirtual.clacso.org.ar/clacso/gt/20100912053709/cultura.pdf>. Recuperado el 15 de febrero de 2020.

MELLADO, Vicente. Concepciones y prácticas de aula de profesores de ciencias, en formación inicial de primaria y secundaria. Investigación y experiencias didácticas. Revista Enseñanza de las Ciencias, v. 14, n. 3, p. 289-302, 1996.

MIGNOLO, Walter. Historias locales/diseños globales: colonialidad, conocimientos subalternos y pensamiento fronterizo. Madrid: Akal, 2003.

MOLINA, Adela; MARTÍNEZ, Carmen; MOSQUERA, Carlos; MOJICA, Lyda. Diversidad cultural e implicaciones en la enseñanza de las ciencias: reflexiones y avances. Revista Colombiana de Educación, v. 56, p. 103-128, 2009.

MORA, Reynaldo. La diversidad cultural en los procesos de construcción curricular. Revista Educación y Humanismo, p. 64-74, 2009.

MOSQUERA, Carlos Javier. EI cambio en la epistemología y en la práctica docente de profesores universitarios de química. Tésis (Doctorado en Didácticas Especificas) - Universidad de Valencia, España, 2008.

NIETO, Jaime. Ciencias Sociales en América Latina: entre el eurocentrismo y el pensamiento crítico. In: ESPERANZA GÓMEZ., Carlos; GAVIRIA, Mariela. (Eds.). Diversidades y decolonialidad del saber en las Ciencias Sociales y el Trabajo Social. Medellín: Pulso \& Letra Editores, 2014.

NúÑEZ, Isabel. La adaptación del currículo escolar a la diversidad cultural. Organización de Estados Iberoamericanos para la Educación. Revista Iberoamericana de Educación. v. 49, pp. 2-10, 2009.

OLMO, Margarita; HERNÁNDEZ, Caridad. Diversidad cultural y educación: la perspectiva antropológica en el análisis del contexto escolar. Formación de la ciudadanía: las TICs y los nuevos problemas, 2004.

PALERMO, Zulma. Colonización del saber: una violencia invisible. INMANENCIA Revista del hospital interzonal general Agudos Eva Perón, v. 3, n. 2, p. 41-44, 2014.

QUIJANO, Anibal. Coloniality of Power, Ethnocentrism, and Latin America. NEPANTLA, v.1, n. 3, p. 533-580, 2000.

RAEL, María Isabel. Educación y sociedad. Innovación y Experiencias Educativas, Granada, 2009. 
RODRÍGUEZ, Rosa María. Atención a la diversidad cultural en la escuela. Propuestas de intervención socioeducativas. Educación y Futuro, v. 10, p. 21-30, 2004.

SÁNCHEZ-ARTEAGA, Juan Manuel. La racionalidad delirante: el racismo científico en la segunda mitad del siglo XIX. Revista de la Asociación Española de Neuropsiquiatría, v 27, n. 2, p. 111-126, 2007.

SÁNCHEZ-ARTEAGA, Juan; SEPÚLVEDA, Cláudia; EL-HANI, Charbel. Racismo científico, procesos de alterización y enseñanza de ciencias. Magis, v. 6, n. 12, p. 55-67, 2013.

TEJADA, José. La educación en el marco de una sociedad global: algunos principios y nuevas exigencias. Revista de Curriculum y Formación del Profesorado, v. 4, n. 1, p. 13-26, 2001.

URIBE, Marisol. Concepciones y prácticas de profesores de ciencias en formación inicial, en realción al enfoque intercultural en la enseñanza de las ciencias: un estudio en el contexto colombiano. Tésis (Doctorado Interinstitucional en Educación con Énfasis en Educación en Ciencias) - Universidad Distrital Francisco José de Caldas, Bogotá, 2019.

VÁZQUEZ, Ángel; ACEVEDO, José; MANASSERO, María.; ACEVEDO, Pilar. Cuatro paradigmas básicos en la naturaleza de las ciencias. Argumentos de Razón Técnica, v. 4, p. 135-176, 2001.

WALSH, Catherine. Interculturalidad crítica y (de)colonialidad: ensayos desde Abya Yala. Quito: Editorial Abya-Yala, 2012. 